Publicacions Matemàtiques, Vol 36 (1992), 211-250.

\title{
THE $p$-PERIOD OF AN INFINITE GROUP
}

\author{
YINING XIA
}

\begin{abstract}
For $\Gamma$ a group of finite virtual cohomological dimension and a primc $p$, the $p$-periad of $\mathrm{I}^{2}$ is defined to be the least positive integer $d$ such that Farrell cohomology groups $\hat{H}^{i}(\Gamma ; M)$ and $\hat{H}^{i+d}(\Gamma ; M)$ have naturally isomorphic $p$-primary components for all integers $i$ and $Z \mathrm{P}$-modules $M$.

We generalize a result of Swan on the p-period of a finite $p$-periodic group to a p-periodic infinite group, i.e., we prove that the p-period of a p-periodic group $I$ of finite vod is $2 L C M(|N(\langle x\rangle) / C(\langle x\rangle)|)$ if the $\Gamma$ has a finite quoticnt whose a $p$ Sylow subgroup is elementary abelian or cyclic, and the kernel is torsion free, where $N(-)$ and $C(-)$ denote normalizer and centralizer, $(x)$ ranges over all conjugacy classes of $Z / p$ subgroups. We apply this result to the computation of the p-period of a p-periodic mapping class group. Also, we give an example to illustrate this formula is false without our assumption.
\end{abstract}

For $\Gamma$ a group of virtual finite cohomological dimension $(v c d)$ and a prime $p$, the $p$-period of $\Gamma$ is defined to be the least positive integer $d$ such that the Farrell cohomology groups $\hat{H}^{i}(\Gamma ; M)$ and $\hat{H}^{i+d}(\Gamma ; M)$ have natually isomorphic $p$-primary components for all $i \in Z$ and $Z \Gamma$-modules $M[3]$.

The following classical result for a finite group $G$ was showed by Swan in 1960 [9].

Theorem (Swan).

a) If a 2-Sylou subgroup of $G$ is cyclic $(\neq\{1\})$, the 2-period of $G$ is 2. If a 2-Sylow subgroup of $G$ is a (generalized) quaternion group, the 2-period of $G$ is 4 .

b) Suppose $p$ an odd prime and a p-Sylow subgroup of the finite group $G$ is cyclic $(\neq\{1\})$. Let $S_{p}$ denote the $p$-Sylow subgroup and $A_{p}$ the group of automorphisms of $S_{p}$ induced by inner automorphism of $G$. Then the p-period of $G$ is twice the order of $A_{p}$. 


\section{Remark.}

The group $A_{p}$ above is isomorphic to $N\left(S_{p}\right) / C\left(S_{p}\right)$, where $N(-)$ and $C(-)$ denote the normalizer and centralizer of $S_{p}$ in $G$.

It is very natural to ask a question: If $\Gamma$ is a $p$-periodic group of finite vcd, is a similar result still true? In other words, is it possible to describe the $p$-period of a $p$-periodic group $\Gamma$ of finite $v c d$ by an algebraic non-homological invariant of the group $\Gamma$ itself?

In this paper, we generalize the result of Swan for a finite group to a $p$ periodic group $\Gamma$ of finite $v c d$ which has a finite quotient whose a $p$-Sylow subgroup is elementary abelian or cyclic, and the kernel is torsion-free, i.e., we prove that the $p$-period of a $p$-periodic group $\Gamma$ of finite $v c d$ is twice the least common multiple of $\{|N(\langle x\rangle) / C(\langle x\rangle)|\}$ in these two cases, where $\langle x\rangle$ ranges over all conjugacy classes of $Z / p$ subgroups of $\Gamma$. On the other hand, we give a group $\Gamma_{0}$ of finite vcd whose only finite subgroup is a $Z / 2$, but the 2-period of $\Gamma_{0}$ is greater than $2|N(Z / 2) / C(Z / 2)|$. Finally, an application will be made for calculating the p-period of a mapping class group.

The following four theorems are our main results of this paper.

Theorem 1. Assume that $\Gamma$ is p-periodic. If $\Gamma$ has a normal subgroup of finite cohomological dimension so that the associated.quoticnt is a finite group whose a p-Sylow subgroup is elementary abelian, then the p-period of $\Gamma$ is twice the least common multiple of $\{|N(\langle x\rangle) / C(\langle x\rangle)|\}$, where $\langle x\rangle$ ranges over all conjugacy classes of $Z / p$ subgroups of $\Gamma$.

Theorem 2. Let $\Gamma$ be a group which has a normal subgroup of finite cohomological dimension so that the associated quotient is a finite group whose a p-Sylow subgroup is cyclic, then the p-period of $\Gamma$ is twice the least common multiple of $\{|N(\langle x\rangle) / C(\langle x\rangle)|\}$, where $\langle x\rangle$ ranges over all conjugacy classes of $Z / p$ subgroups of $\Gamma$.

Theorem 3. There is a group $\Gamma_{0}$ of finite vcd whose only finite subgroup is a $Z / 2$, but the 2-period is greater than $2|N(Z / 2) / C(Z / 2)|$.

Theorem 4. If the mapping class group $\Gamma_{g}$ is a p-periodic group and $g<p(p-1) / 2$, then the $p$-period of $\Gamma_{g}$ is $2 L C M\left\{\operatorname{gcd}\left(p-1, b_{i}\right)\right\}$, where $b_{i} \in B_{g, p}$ (cf. section 3$)$.

The rest of this paper is organized as follows. In section 1 , we prove Theorems 1 and 2 . In section 2 , we provide an example illustrating Theorem 3. Finally in section 3 , we give a formula for the calculation of the $p$-period of a $p$-periodic mapping class group $\Gamma_{p}$. 


\section{Proof of Theorems 1 and 2}

Lemma 1.1. Let $H=\left\langle x, y / x^{p}=1, y x y^{-1}=x^{r}\right\rangle$, where $q=0$ or $q \neq 0 \bmod (p)$. If $d$ is the minimal positive integer such that $r^{d}=1$ $\bmod (p)$, then the p-period of $H$ equals $2 d$.

Proof: If $q \neq 0, H$ is a finite group, the proof is immediate by Swan Theorem. Otherwise, if $q=0, H$ is infinite and we look at the short exact sequence $1 \rightarrow Z / p \rightarrow H \rightarrow Z \rightarrow 1$. The spectral sequence of Farrell cohomology associated to the exact sequence converges in the following way: $E_{2}^{i, 3}=H^{i}\left(Z ; \hat{H}^{j}(Z / p ; Z)\right) \rightarrow \hat{H}^{i+j}(H ; Z)[2]$. This spectral sequence collapses sincc $H^{i}\left(Z ; \hat{H}^{j}(Z / p ; Z)\right)=0$ when $i<0$ or $i>1$. Therefore, $1 \rightarrow \hat{H}^{n-1}(Z / p ; Z)_{Z} \rightarrow \hat{H}^{n}(H ; Z) \rightarrow \hat{H}^{n}(Z / p ; Z)^{Z} \rightarrow 1$ is an exact sequence. By looking at the $Z$ action on the subgroup $Z / p, u^{d} \in$ $\hat{H}^{2 d}(Z / p ; Z)$ is an invariant element of the $Z$ action on $\hat{H}^{2 d}(Z / p ; Z)$. Here $u$ is a generator of $\hat{H}^{2}(Z / p, Z)$. Therefore, there exists an element $h \in \hat{H}^{2 d}(H ; Z)$ such that $\operatorname{Res}(h)=u^{d} \neq 0$ on $\hat{H}^{2 d}(Z / p ; Z)$. By Brown-Venkov theorem $[2]$ and $\hat{H}^{2 k d}(H ; Z)=Z / p, \hat{H}^{2 k d+1}(H ; Z)=$ $Z / p, \hat{H}^{i}(H ; Z)=0$ for other i's, the $p$-period of $H$ is $2 d$.

Lemma 1.2. Let $Z / p$ be a normal subgroup of a group $\Gamma$ of finite $v c d$, and let $M$ be a finite quotient of $\Gamma$ with torsion free kemel. Then $\Gamma / C_{\Gamma}(Z / p)=N_{\Gamma}(Z / p) / C_{\Gamma}(Z / p)=N_{M}(Z / p) / C_{M}(Z / p)=M / C_{M}(Z / p)$. Here we sitll use $Z / p$ to stand for the image of $Z / p$ in $M$.

Proof: Let $p r: \Gamma \rightarrow M$ be the natural projection map. The map pr maps $N_{\Gamma}(Z / p)$ onto $N_{M}(Z / p)$ and $C_{\Gamma}(Z / p)$ to $C_{M}(Z / p)$, so induced map $p r_{*}: N_{\Gamma}(Z / p) / C_{\Gamma}(Z / p) \rightarrow N_{M}(Z / p) / C_{M}(Z / p)$ is a welldefined surjective homomorphism. Let $\langle x\rangle=Z / p$, if $y x y^{-1}=x^{r}$, then $p r(y) x p r(y)^{-1}=x^{r}$, i.e., $p r_{*}$ is an injective.

Lemma 1.3. Suppose a group $M$ contains a cyclic subgroup $Z / p^{n} \supset$ $Z / p$ and $\left|N\left(Z / p^{n}\right) / C\left(Z / p^{n}\right)\right|$ is prime to $p$, then the homomorphism induced by inclusion $i_{*}: N\left(Z / p^{n}\right) / C\left(Z / p^{n}\right) \rightarrow N(Z / p) / C(Z / p)$ is injective.

Proof: Notice $N(Z / p) \supset N\left(Z / p^{n}\right)$ and the inclusion $i$ maps $C\left(Z / p^{n}\right)$ to $C(Z / p)$, i.e., the induced map by inclusion $i_{*}: N\left(Z / p^{n}\right) / C\left(Z / p^{n}\right) \rightarrow$ $N(Z / p) / C(Z / p)$ is a well-defined homomorphism. Now let $\langle x\rangle=Z / p^{n}$, then $\left\langle x^{p^{n-1}}\right\rangle=Z / p$, if $y \in C(Z / p), y x y^{-1}=x^{k}$, then $y x^{p^{n-1}} y^{-1}=$ $x^{k p^{n-1}}=x^{p^{n-1}}$, so $(k-1) p^{n-1}=0 \bmod \left(p^{n}\right)$, i.e., $k=1 \bmod (p)$. Let $k=$ $A p^{m}+1, A$ is prime to $p$ and $1 \leq m<n, k^{d}=1 \bmod \left(p^{n}\right), d$ divides $p-1$ 
by assumption. Hence $k^{d}=\left(A p^{m}+1\right)^{d}=B+A d p^{m}+1=1 \bmod \left(p^{n}\right)$, where $p^{2 \pi t}$ divides $B$. This implies $A d=0 \bmod (p)$, a contradiction unless $A=0$.

Lemma 1.4 (Swan) [9]. Suppose the p-Sylow subgroup $S_{p}$ of a finite group $M$ is abelian. Let $A_{p}$ be the group of automorphisms of $S_{p}$ induced by inner automorphisms of $M$. Then an element $a \in H^{i}\left(S_{p} ; Z\right)$ is stable if and only if it is fixed under the action of $A_{p}$ on $H^{i}\left(S_{p} ; Z\right)$.

\section{Proof: See [9].}

Proof of Theorem 1: A theorem of Brown [3, p. 293] states that if $\Gamma$ is $p$-periodic, then $\hat{H}^{*}(\Gamma ; Z)_{(p)}=\prod_{P_{j} \in S} \hat{H}^{*}\left(N\left(P_{i}\right) ; Z\right)_{(p)}$, where $S$ is the set of all conjugacy classcs of $Z / p$ of $\Gamma$. Therefore, the $p$-period of $\Gamma$ is the least common multiple of the $p$-periods of $N_{\mathrm{r}}\left(P_{i}\right)$.

1) Lower bound. Let $\left|N_{\Gamma}\left(P_{i}\right) / C_{\Gamma}\left(P_{2}\right)\right|=d_{i},\langle x\rangle=P_{i}$. There exists $y \in \Gamma$, such that $y x y^{-1}=x^{r}, r^{d_{i}}=1 \bmod (p)$. Let $H=\langle x, y\rangle$ be a subgroup of $\Gamma$ generated by elements $x$ and $y$. Then the $p$-period of $H$ is $2 d_{i}$ by Lemma 1.1, i.e., the p-period of $N_{\Gamma}\left(P_{i}\right)$ is a multiple of $2 d_{i}$.

2) Upper bound. Let $p r: \Gamma \rightarrow M$ be a projection onto the finite quotient $M$ whose a $p$-Sylow subgroup is elcmentary abelian, and $p r_{i}: N_{\mathrm{l}}\left(P_{i}\right) \rightarrow M_{i}$ be the restriction map of $p r_{\text {, where }} M_{i}$ is the image of $p r_{i}$. Then $M_{i}=\operatorname{Im} N_{\Gamma}\left(P_{i}\right)=N_{M_{i}}\left(P_{i}\right)$ normalizes $P_{i}\left(P_{i}\right.$ also denotes the image of $P_{i}$ ), the group $A_{p}$ of automorphisms of $S_{p}$ induced by inner automorphisms of $M_{i}$ maps $P_{i}$ to itself.

Lct $u \in H^{2}\left(S_{p} ; Z\right)=\operatorname{Hom}\left(P_{i} \times Z / p \times \ldots \ldots Z / p, C^{*}\right)$ be a cohomology element such that $u(x) \neq 1$ and $u(y)=1$ if $\langle x\rangle=P_{i},\langle y\rangle=Z / p$, where $C^{*}$ is the multiple group of nonzcro complex numbers. Then $\operatorname{Res}(u) \neq 0$ in $H^{2}\left(P_{i} ; Z\right)$. Now we claim that $u^{d_{2}} \in H^{2 d_{i}}\left(S_{p} ; Z\right)$ is a stable element for $S_{p}$ in $M_{i}$. In fact, $d_{i}=\left|N_{M_{i}}\left(P_{i}\right) / C_{M_{i}}\left(P_{i}\right)\right|$ by Lemma 1.2 , and $A_{p}$ fixes the element $u^{d_{2}} \in H^{2 d_{i}}\left(S_{p} ; Z\right)$ since $N_{M_{i}}\left(P_{i}\right) / C_{M_{i}}\left(P_{i}\right)$ fixes the element $u^{d_{i}}$. By Lemma $1.4[9], u^{d_{2}}$ is a stablc clement for $S_{p}$ in $M_{i}$, i.c., there exists an element $v \in H^{2 d_{i}}\left(M_{i} ; Z\right)$ such that $\operatorname{Re} s_{P_{2}}^{M i}(v)=\operatorname{Res} s_{P_{i}}^{S_{p}}\left(u^{d_{i}}\right)=\left[\operatorname{Re} s_{P_{i}}^{S p}(u)\right]^{d_{i}} \neq 0$. If wc apply the canonical homomorphism $g^{*}$ from ordinary cohomology to Farrell cohomology [3, p. 278] we have $\operatorname{Re} s_{P_{i}}^{M_{i}}\left(g^{*}(v)\right)=\operatorname{Re} s_{P_{i}}^{S_{p}}\left(g^{*}\left(u^{d_{i}}\right)\right)=\operatorname{Re} S_{P_{i}}^{S p_{p}}\left(g^{*}(u)\right)^{d_{i}} \neq 0$, i.e., there exists an element $\operatorname{pr}_{i}^{*} g^{*}(v) \in \hat{H}^{2 d_{i}}\left(N_{\Gamma}\left(P_{i}\right) ; Z\right)$ such that $\operatorname{Res}_{P i}^{N\left(P_{i j}\right)}\left(\operatorname{pr}_{i}^{*} g^{*}(v)\right) \neq 0$ in $\hat{H}^{2 d_{i}}\left(P_{i} ; Z\right)$, by Brown-Venkov theorem [2] and the fact that $N_{\Gamma}\left(P_{i}\right)$ has only one order $p$ subgroup, the $p$-period of 
$N_{\Gamma}\left(P_{i}\right)$ divides $2 d_{i}$. Sec following diagram.

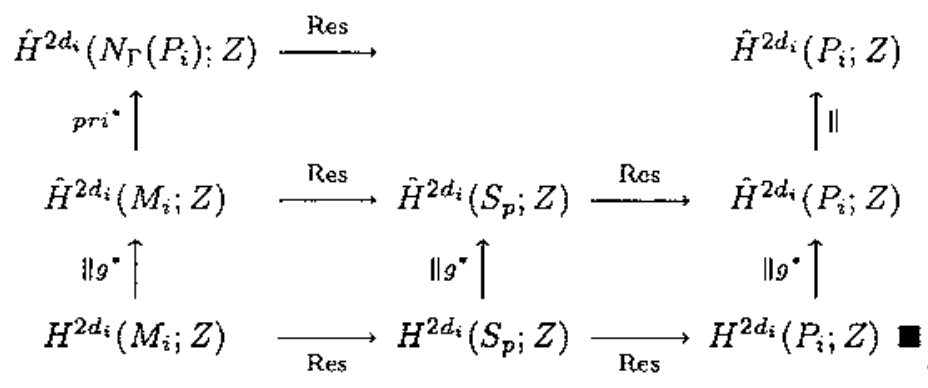

Proof of Theorem 2: is basically a similar argument except for the upper bound part. In fact, if $\Gamma$ has a finite $p$-pcriodic quotient $M$ with torsion free kernel, then $\Gamma$ is $p$-periodic and the $p$-period of $\Gamma$ divides the $p$-period of $M$. This is because the infation map $\hat{H}^{*}(M) \rightarrow \hat{H}^{*}(\Gamma)$ maps an invertible element of $\hat{H}^{*}(M)$ to an invertible element of $\hat{H}^{*}(\Gamma)$. Using Swan Theorem, we obtain that the $p$-period of $N_{1}\left(P_{i}\right)$ divides the $p$-period of $M_{i}$, which is $2\left|N_{M_{2}}\left(Z / p^{n}\right) / C_{M_{i}}\left(Z / p^{n}\right)\right|$. Also, by Lemma 1.3, the number $2\left|N_{M_{i}}\left(Z / p^{n}\right) / C_{M_{i}}\left(Z / p^{n}\right)\right|$ divides $2\left|N_{M_{i}}\left(P_{i}\right) / C_{M_{i}}\left(P_{i}\right)\right|=$ $2\left|N_{\Gamma}\left(P_{i}\right) / C_{\Gamma}\left(P_{i}\right)\right|$.

\section{An example}

Lemma 1.3, Lemma 1.1 and Swan Theorem imply that the equality $\left|N\left(S_{p}\right) / C\left(S_{p}\right)\right|=|N(Z / p) / C(Z / p)|$ holds in the case of a finite group $G$ whose a $p$-Sylow subgroup is cyclic, here $Z / p$ is the order $p$ subgroup of $S_{p}$. Therefore, Theorems 1 and 2 are generalizations of Swan Theorem.

In the case of a group $\Gamma$ of finite vcd, in general, $\left|N\left(S_{p}\right) / C\left(S_{p}\right)\right| \neq$ $|N(Z / p) / C(Z / p)|$ even if all maximal $p$-subgroups $S_{p}$ of $\Gamma$ are cyclic. For example, let $\Gamma^{*}=\left\langle x, y \mid x^{p^{2}}=1, y x y^{-1}=x^{p+1}\right\rangle$, and $d$ is the minimal positive integer such that $(p+1)^{d}=1 \bmod \left(p^{2}\right)$. Then $|N(\langle x\rangle) / C(\langle x\rangle)|=$ $d=p$, but $\left|N\left(\left\langle x^{p}\right\rangle\right) / C\left(\left\langle x^{p}\right\rangle\right)\right|=1$. A similar argument to Lerrma 1.1 shows the $p$-period of $\Gamma^{*}$ above equals $2 p$. This trivial example shows that the $p$-period of an infinite group $\Gamma$ can not be only described in the form $2 L C M\{|N(Z / p) / C(Z / p)|\}$ in general.

The example $\Gamma^{*}$ above could lead us to thirk that the $p$-period of a $p$ periodic group $\Gamma$ equals $2 L C M\{|N(C(p)) / C(C(p))|\}$, wherc $C(p)$ ranges over all conjugacy classes of maximal $p$-cyclic subgroups of $\Gamma$. Recall in the case of a finite group $G$, Swan Theorem can be also stated in the different form: the $p$-pcriod of $G$ equals $2|N(C(p)) / C(C(p))|$ (including the case $p=2$ ), where $C(p)$ is a maximal $p$-cyclic subgroup of $G$. 
Unfortunately, the next example shows that this is not true.

Example. Let $\Gamma_{n, m}$ denote the congruence subgroup of $S L(n, Z)$ of level $m$, i.e., the kernel of the surjective homornorphism $r_{m}: S L(n, Z) \rightarrow$ $S L(n, Z / m)$ induced by the reduction $\bmod (m)$ ( $m$ may not be prime). It is well-known that the group $\Gamma_{n, m}$ is always torsion free when $n \geq 1$ and $m \geq 3$. A result of Charney [4] states that the group $\Gamma_{n, p}$ is cohomology stable with $Z / 2$ coefficient for any odd prime $p$. Define $\Gamma_{p}=\lim _{n} \Gamma_{n, p}$, then $H^{i}\left(\Gamma_{n, p} ; Z / 2\right)=H^{i}\left(\Gamma_{p} ; Z / 2\right)$ for $n \geq 2 i+5$.

Let $G L(Z)$ be the infinite general linear group of $Z$ and $w_{i} \in$ $H^{i}(G L(Z) ; Z / 2)$ the i-th Sticfcl-Whitney class of the inclusion $G L(Z) \rightarrow$ $G L(R)$ for $i \geq 1$. We still denote by $w_{i}$ the image of $w_{i}$ under the restriction $H^{i}(G L(Z) ; Z / 2) \rightarrow H^{i}(S L(Z) ; Z / 2) \rightarrow H^{i}\left(\Gamma_{m} ; Z / 2\right)$.

The calculation in [1] by Arlettaz gives following results: for any odd prime $p$

a) $w_{1}\left(\Gamma_{p}\right)=0$

b) $w_{2}\left(\Gamma_{p}\right) \neq 0$

c) $w_{3}\left(\Gamma_{p}\right)=0$ if and only if $p=7 \bmod (8)$.

Also, we know from $W u$ formula for the Steenrod square $S q^{1}\left(w_{2}\right)=$ $w_{1} w_{2}+w_{0} w_{3}=w_{3}$ in $H^{3}\left(\Gamma_{p} ; Z / 2\right)$. Again, denote by $w_{i}$ the image of $w_{i}$ under the restriction $H^{i}\left(\Gamma_{5} ; Z / 2\right) \rightarrow H^{i}\left(\Gamma_{11,5}\right)$. Combining both results of Charney and Arlettaz above, we have $w_{1}=0, w_{2} \neq 0$ and $S q^{1}\left(w_{2}\right)=w_{3} \neq 0$ in $H^{*}\left(\Gamma_{11,5} ; Z / 2\right)$ (in fact, these are all true for $H^{*}\left(\Gamma_{n, 5} ; Z / 2\right)$ as long as $n \geq 11$.)

Let $\Gamma_{0}$ denote the group of the extension $I \rightarrow Z / 2 \rightarrow \Gamma_{0} \rightarrow$ $\Gamma_{11,5} \rightarrow 1$ which corresponds to the non-trivial cohomology element $w_{2} \in H^{2}\left(\Gamma_{11,5} ; Z / 2\right)$. Obviously, the group $\Gamma_{0}$ contains only one $2-$ subgroup $Z / 2$, and the extension is central. Next, we check that the group $\Gamma_{0}$ is of finite $v c d$, then show that the 2-period of $\Gamma_{0}$ is greater than 2.

Consider the following commutative diagram, where all maps $R_{1}, R_{2}$, $R_{3}$ and $R_{4}$ are restriction maps.

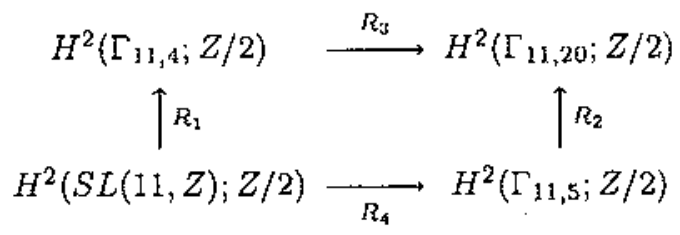

In fact, the map $R_{1}=0$ is a special case of the result by Milison [7; $\mathrm{p}$. 85] which states that for any $n \geq 3$ the map $r^{*}: H^{2}(S L(n, Z / 4) ; Z / 2) \rightarrow$ $H^{2}(S L(n, Z), Z / 2)$ induced by the reduction $\bmod (4)$ is an isomorphism. 
Thus, we obtain the nontrivial second Stiefel-Whitney class $w_{2}$ in $H^{2}\left(\Gamma_{11,5} ; Z / 2\right)$, but the restriction of $w_{2}$ into the cohomology of the finitc index subgroup $H^{2}\left(\Gamma_{11,20} ; Z / 2\right)$ is 0 . This actually proves that the group $\Gamma_{0}$ is finite $v c d$ and the $v c d\left(\Gamma_{0}\right)=c d\left(\Gamma_{11,20}\right)=v c d(S L(11, Z))=55[3$, p. 229].

In order to find a lower bound on the 2-period of $\Gamma_{0}$, consider two spectral sequences as follows:

1. The Lyndon-Hochschild-Serre spectral sequence of the group extension $1 \rightarrow Z / 2 \rightarrow \Gamma_{0} \rightarrow \Gamma_{11,5} \rightarrow 1$ with $Z / 2$ coefficient. This takes the form $E_{2}^{i, j}=H^{i}\left(\Gamma_{11,5} ; H^{j}(Z / 2 ; Z / 2)\right) \Rightarrow H^{i+j}\left(\Gamma_{0} ; Z / 2\right)$.

2. The Farrell cohomology spectral sequence $[2]$ of the group extension $1 \rightarrow Z / 2 \rightarrow \Gamma_{0} \rightarrow \Gamma_{11,5} \rightarrow 1$ with $Z / 2$ cocfficicnt. This takcs the form $E_{2}^{i, j}=H^{i}\left(\Gamma_{11,5} ; \hat{H}^{j}(Z / 2 ; Z / 2)\right) \Rightarrow \hat{H}^{i+j}\left(\Gamma_{0} ; Z / 2\right)$.

Let $u \in H^{1}(Z / 2 ; Z / 2)$ be the generator of the cohomology ring $H^{*}(Z / 2 ; Z / 2)=F_{2}[u]$, and $d_{2}(u)=w_{2} \in H^{2}\left(\Gamma^{\prime} ; Z / 2\right)$ be the second Stiefel-Whitrey class corresponding to the cxtension $1 \rightarrow Z / 2 \rightarrow \Gamma_{0} \rightarrow$ $\Gamma_{11,5} \rightarrow 1$. Then $u$ is transgressive, $d_{2}(u)=\tau(u)=w_{2}$, where $\tau$ is the transgression. The element $u^{2}=S q^{1}(u)$ is also transgressive $[8, \mathrm{p} .81]$, and $d_{3}\left(u^{2}\right)=\tau\left(u^{2}\right)=\tau\left(S q^{1}(u)\right)=S q^{1}(\tau(u))=S q^{1}\left(w_{2}\right)=w_{3} \neq 0$ in $E_{3}$ because $H^{1}\left(\Gamma_{1,1,5} ; Z / 2\right)$ is trivial.

Consider a commutative diagram involving in both spectral sequences as follows:

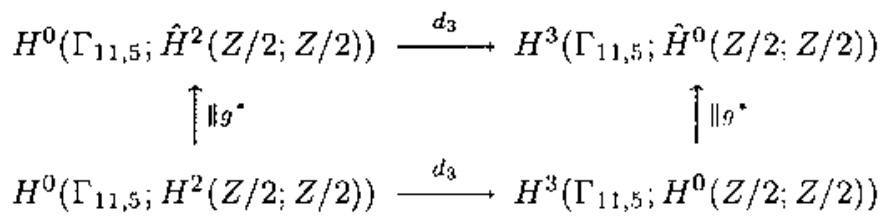

The nontriviality of $d_{3}$ in the second row implies the nontriviality of $d_{3}$ in the first row. This shows Res: $\hat{H}^{2}\left(\Gamma_{0} ; Z / 2\right) \rightarrow \hat{H}^{2}(Z / 2 ; Z / 2)$ is trivial since the map Res factors through $E_{\infty}^{0,2}=0$. Thercfore, there is no invertible clement in $\hat{H}^{2}\left(\Gamma_{0} ; Z / 2\right)$. By the fact that the reduced map $\hat{H}^{2}\left(\Gamma_{0} ; Z\right)_{(2)} \rightarrow \hat{H}^{2}\left(\Gamma_{0} ; Z / 2\right)$ is ring homomorphism, there is no invertible element in $\hat{H}^{2}\left(\Gamma_{0} ; Z\right)_{(2)}$, i.c., the 2-period of $\Gamma_{0}$ is grcater than 2. We have proved our Theorem 3.

\section{The $p$-period of the mapping class group $\Gamma_{g}$}

The $p$-periodicity of the mapping class group is studied in a different paper of the author [11]. As an application of the theorern 1, we obtain the $p$-period of a $p$-periodic mapping class group $\Gamma_{g}$ when $g<p(p-1) / 2$. 
Recall that the mapping class group $\Gamma_{g}$ is defined to be the group of path components of orientation prescrving diffeomorphisms of the closed orientable surface $S_{g}$ of genus $g>1$. Next, we define a set $B_{g_{1} p}$ for surface $S_{g}$ and a prime $p$.

Definition. For $p$ odd, let $2 g-2=m p-i, 0 \leq i \leq p-1$.

$$
\begin{aligned}
& B_{g, p}=\{i, i+p, i+2 p, \ldots \ldots i+([2 g /(p-1)]-m) p\} \text { if } i \neq 1 . \\
& B_{g, p}=\{1+p, 1+2 p, \ldots \ldots 1+([2 g /(p-1)]-m) p\} \text { if } i=1
\end{aligned}
$$

And for $p=2$,

$$
\begin{aligned}
& B_{g, 2}=\{0,4,8, \ldots \ldots 2 g+2\} \text { if } g \text { is odd. } \\
& B_{g, 2}=\{2,6,10, \ldots \ldots 2 g+2\} \text { if } g \text { is even. }
\end{aligned}
$$

\section{Remarks.}

1. The notation $[-]$ here means the intcoger part.

In case $i \neq 1,2 g /(p-1)<\pi n_{1}$ define $B_{g, p}=\emptyset$.

In case $i=1,2 g /(p-1)<m+1$, define $B_{g, p}=0$.

2. It is proved in [11] that the set $B_{9, p}$ is exactly the sct of all possible number of fixed points when an order $p$ diffeomorphism acts on the surface $S_{3}$.

Lemma 3.1. For the mapping class group $\Gamma_{y}$, there is a formula $L C M\left\{\mid(N(\langle x\rangle) / C(\langle x\rangle) \mid\}=L C M\left\{\operatorname{gcd}\left(p-1, b_{i}\right)\right\}\right.$, where $\langle x\rangle$ ringes over all conjugacy. classes of $Z / p$ in $\Gamma_{g}, b_{i}$ ranges over all $b_{i} \in B_{g, p}$.

Proof: 1) Assume $[N(\langle x\rangle) / C(\langle x\rangle)]=d$. Then there exists an integer $r$ such that $x \approx x^{r} \approx \cdots \cdots \approx x^{r^{d, 1}}\left(\approx\right.$ means "is conjugate to" in $\left.\Gamma_{g}\right)$ so that $d$ is the minimal positive integer satisfying $r^{d}=1 \bmod (p)$. The $d$ divides $p-1$ obviously. Let $b$ be the number of fixed points of the $x$ action on $S_{g}, \sigma(x)=\left(\beta_{1}, \beta_{2}, \ldots \ldots \beta_{b}\right)$ the fixed point datum, where $\beta_{i} \in Z / p-\{0\}$ (cf. $[\mathbf{1 0}]$ ).

Let us define a perrmutation $r^{*}$ on the ordered $b_{i}$-tuple $\left(\beta_{1}, \beta_{2}, \ldots, \beta_{b_{i}}\right)$. Set $r^{*}\left(\beta_{1}, \beta_{2}, \ldots \ldots \beta_{b_{i}}\right)=\left(r \beta_{1}, r \beta_{2}, \ldots \ldots r \beta_{b_{i}}\right),\left(r^{*}\right)^{2}=\left(r^{2}\right)^{*} \ldots$ $\ldots\left(r^{*}\right)^{d-1}=\left(r^{d-1}\right)^{*}$. It is well-defined since $\sigma(x)=\sigma\left(x^{r^{2}}\right)=$ $\cdots \cdots=\sigma\left(x^{r^{d-1}}\right)$ as an unordered b-tuples [12]. We can decompose $r^{*}=\left(\beta_{i_{1}}, \beta_{i_{2}}, \ldots \ldots \beta_{i_{s}}\right)\left(\beta_{j_{1}}, \beta_{j_{2}}, \ldots \ldots \beta_{j_{t}}\right) \ldots \ldots\left(\beta_{k_{1}}, \beta_{k_{2}}, \ldots \ldots \beta_{k_{u}}\right)$, a product of cyclic permutations. Notice that permutations $r^{*},\left(r^{*}\right)^{2}, \ldots$ $\ldots\left(r^{*}\right)^{d-1}$ do not have fixed points. Otherwise, there exists $\beta_{i}$ such that $r j \beta_{i}=\beta_{\imath} \bmod (p), 1 \leq j \leq d-1$. This forces $r j=1 \bmod (p)$, a contradiction. But, of course, $\left(r^{*}\right)^{d}=\left(r^{d}\right)^{*}=$ Id. These imply 
$s=t=\cdots \cdots=u=d$, i.e., the number $|N(\langle x\rangle) / C(\langle x\rangle)|=d$ divides the number $b_{i}$ of fixed points of the $x$ action on the surface $S_{q}$. We have showed that $L C M\{|N(\langle x\rangle) / C(\langle x\rangle)|\}$ divides $L C M\left\{\operatorname{gcd}\left(p-1, b_{i}\right)\right\}$, where $\langle x\rangle$ ranges over all conjugacy classes of $Z / p$ in $\Gamma_{g}, b_{i}$ ranges over all $b_{i} \in B_{g, p}$.

2) Conversely, assume $\operatorname{gcd}\left(p-1, b_{i}\right)=d$. Then there is a $\bmod (p)$ integer $r$ so that $d$ is a minimal positivc intcger satisfying $r^{d}=1 \bmod (p)$.

Case 1. $b_{i} \neq 0$. If $d \neq 1$, then $r \neq 1$. Consider the unordered $b_{2}$-tuples $\sigma=\left(1, r, r^{2}, \ldots \ldots r^{d-1}, 1, r, r^{2}, \ldots \ldots r^{d-1}, \ldots \ldots 1, r, r^{2}, \ldots \ldots, r^{d-1}\right)$. Since $\left(b_{i} / d\right)\left(1+r+r^{2}+\ldots \ldots r^{t-1}\right)=0 \bmod (p)$. There cxists an element $x \in \Gamma_{g}, x^{p}=1$, and the it's representive fixed point datum $\sigma(x)$ is $\sigma$, i.c., the unordered $b_{i}$-tuples $\sigma$ can be realized as a fixed point datum of an order $p$ element in $\Gamma_{g}[6]$. Obviously, $\sigma(x)=\sigma\left(x^{r}\right)=\sigma\left(x^{r^{2}}\right)=\cdots \cdots=$ $\sigma\left(x^{r^{d-1}}\right)$ or $x \approx x^{r} \approx x^{r^{2}} \approx \cdots \cdots \approx x^{r^{d}}$ ' in $\Gamma_{y}$. This implies that the number $d$ divides the order $|N(\langle x\rangle) / C(\langle x\rangle)|$. If $\operatorname{gcd}\left(p-1, b_{i}\right)=d=1$, for any order $p$ element $x$ in $\Gamma_{g}$ with the mumber of fixcd points $b_{i}$, obviously 1 divides $|N(\langle x\rangle) / C(\langle x\rangle)|$.

Case 2. $b_{i}=0$ ). On the one hand, we have $\operatorname{ged}\left(p-1, b_{i}\right)=p-1$. On the other hand, the $x$ acts on $S_{9}$ freely. All order $p$ froc actions are conjugate by $[\mathbf{5}]$, this implies $|N(\langle x\rangle) / C(\langle x\rangle)|=p-1$.

So, $L C M\left\{\operatorname{gcd}\left(p-1, b_{i}\right)\right\}$ divides $L C M\{|N(\langle x\rangle) / C(\langle x\rangle)|\}$.

Proof of Theorem 4: Let $\mu: \Gamma_{g} \rightarrow S p(2 g, Z)$ be the canonical homology representation and $p: S p(2 g, Z) \rightarrow S p\left(2 q, F_{q}\right)$ be the reduction map. Here $q$ can be chosen a primitive root of $\bmod (p)$ such that $q \geq 3$, and $q^{p-1}$ is not congrucnt to $1 \bmod \left(p^{2}\right)$ (by the Dirichlet theorem).

Now $\operatorname{Ker}(p \mu)=N$ is a torsion free, normal, finite index subgroup of $\Gamma_{g}$ and a $p$-Sylow subgroup of the finite quoticnt $\Gamma_{g} / N=S_{p}\left(2 g, F_{q}\right)$ is elementary abelian if $2 g<p(p-1)$. Then we can use Thoorcm 1 and Lernma 3.1 to finish the proof.

A list of the $p$-pcriod of a $p$-pcriodic mapping class group $\Gamma_{g}$ can be also found in the Appendix $C$ of the author's thesis [12].

Acknowledgement. The most parts of this paper are from the Ohio State University thesis [13] which is written by the anthor under the: direction of Professor H. Glover and Professor G. Mislin. The author wishes to express the most sincere thanks to Professor H. Glover and Professor G. Mislin for their excellent guidance and inspiration.

\section{References}

1. Arimtraz, D., Sur les classes de Sticfel-Whitney des sous-groupes 
de congruence, C.R. Acad. Sci. Paris Ser. I Math. 303 (1986), $571-574$.

2. Brown, K.S., Groups of virtually finite dimension, Proc. of Sept, 1977, Durham conference on homological and combinatorial techniques in group theory, $27 \cdots 70$.

3. Brown, K.S., "Cohomology of groups," Graduate Texts in Math. 87, Springer-Verlag, 1982.

4. Charney, R., On the problem of homology stability for congruence subgroups, Comm. in Algebra 12 (1984), 2081-2123.

5. Edmonds, A., Surface symmetry I, Mich. Math. J. 29 (1982), 171-183.

6. EwiNG, J., "Antomorphisms of surfaces ond class numbers: An illustration of the $G$-index theorem." London Math. Soc. Lecture note series 86, 1983, pp. 120-127.

7. Millson, J., Real vector bundles with discrete structure group, Topology 18 (1979), 83-89.

8. Mosher, R. AND Tavgora, M., "Cohomology operations and Applications in homotopy theory." Harper and Row, Publishers.

9. SWAs', R., The p-period of a finitc group, Ihinois J. Math. 4 (1960), 341-346.

10. SYMONDs, P., The cohomology representation of an action of $C_{p}$ on a surface, Trans. Amer, Math. Sor. 306 (1988), 389-400.

11. XiA, Y., The $p$-periodicity of the rnapping class group and the estimate of its $p$-period, Proc. of AMS, to appear.

12. $\mathrm{X}_{\mathrm{IA}}, \mathrm{Y}$, Farrell-Tate cohomology of the mapping class group, Thesis, The Ohio State University.

Department of Mathematies The Ohio State University

Columbus, Ohio 43210-1174

U.S.A.

Primera versió rebuda el 21 de Juny de 1991, dorrera versió rebuda el 13 de Febrer de 1992 